\title{
Longitudinal Measurement of Pleural Fluid Biochemistry and Cytokines in Malignant Pleural Effusions
}

\section{Authors:}

* Rajesh $\underline{\text { Thomas }}^{1,2,3}$ MBBS, FRACP rajesh.thomas@health.wa.gov.au

* Hui Min $\underline{\text { Cheah }}^{2,3}$ BSc hui.cheah@uwa.edu.au

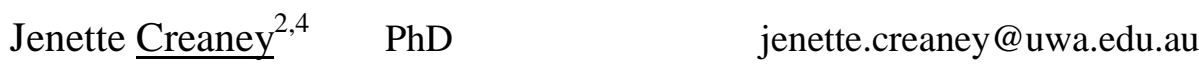

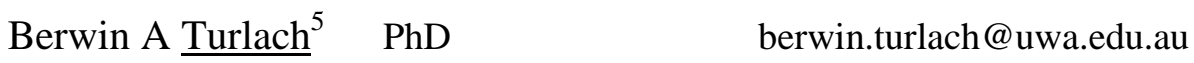

Y C Gary $\underline{L e}^{1,2,3}$ MBChB PhD FRCP FCCP FRCP gary.lee@uwa.edu.au

* The authors contributed equally to the work.

\section{Affiliations:}

${ }^{1}$ Dept of Respiratory Medicine, Sir Charles Gairdner Hospital;

${ }^{2}$ School of Medicine \& Pharmacology, University of Western Australia;

${ }^{3}$ Pleural Medicine Unit, Institute of Respiratory Health;

${ }^{4}$ National Centre for Asbestos Related Diseases;

${ }^{5}$ Centre for Applied Statistics and School of Mathematics and Statistics, University of Western Australia

Perth, AUSTRALIA

Contributions: Guarantor - YCGL; Conception \& Design - RT, YCGL;

Clinical data collection - RT; Bio-banking \& Sample processing - HMC, JC;

Cytokine measurements - HMC; Statistical Analyses - RT, BAT, YCGL;

Manuscript drafting and revision - all authors; Final approval - all authors.

Correspondence: Prof Y C Gary LEE, Centre for Asthma, Allergy \& Respiratory Research, University of Western Australia, 5/F, QQ Blk, QE II Med Ctr, Perth WA 6009, Australia. Tel: +61 861510913. 


\section{Funding:}

Prof Lee is a National Health \& Medical Research Council (NH\&MRC) Career Development Fellow and receives research project grant funding from the NH\&MRC, New South Wales Dust Disease Board, Sir Charles Gairdner Research Advisory Committee, Westcare and the Cancer Council of Western Australia.

Dr Thomas has received research scholarship support from NH\&MRC, WA Cancer and Palliative Care Network, and Institute of Respiratory Health.

Dr Creaney receives research project grant funding from the NH\&MRC, New South Wales Dust Disease Board, Sir Charles Gairdner Research Advisory Committee, and the Cancer Council of Western Australia.

Ms Cheah has received post-graduate research scholarships from the University of Western Australia and the Institute of Respiratory Health.

\section{Conflict of Interest:}

No authors have a conflict of interest.

\section{Word count (Text): 2503}

Keywords: Malignant pleural effusion, pleural fluid, mesothelioma, protein, LDH, pH, glucose, cytokines, MCP-1 


\section{ABSTRACT}

Background: Malignant pleural effusion (MPE) is common. Existing literature on pleural fluid compositions are restricted to cross-sectional sampling with little information on their longitudinal changes with disease progression. Indwelling pleural catheters (IPC) provide the unique opportunity for repeated sampling and longitudinal evaluation of MPE, which may provide insight on tumor pathobiology.

Methods: 638 MPE samples were collected from 103 patients managed with IPCs over 95 (median, range 0-735) days and analysed for levels of protein, $\mathrm{pH}, \mathrm{LDH}$ and glucose. Peripheral blood was quantified for haematocrit, platelets, leukocytes, protein and albumin. Cytokine levels (monocyte chemotactic protein [MCP]-1, vascular endothelial growth factor, interleukin [IL]-6, $-8,-10$, tumor necrosis factor- $\alpha$ and interferon- $\gamma$ ) were determined in 298 samples from 35 randomly selected patients. Longitudinal changes of all parameters measured were analysed using a linear mixed model.

Results: Significant decreases were observed over time in pleural fluid protein by $8 \mathrm{~g} / \mathrm{L} / 100$ days (SE 1.32; $p<0.0001)$ and $\mathrm{pH}(0.04 / 100$ days, SE $0.02 ; p=0.0203$ ), accompanied by a non-significant rise of LDH. The pleural fluid to serum protein ratio decreased by $0.06 / 100$ days (SE $0.02 ; p=0.04$ ). MPEs from mesothelioma $(n=63)$ had lower pleural fluid glucose $(p=0.0104)$ at baseline and a faster rate of decline in glucose $(p=0.0423)$ when compared with non-mesothelioma effusions $(n=38)$. A progressive rise in pleural fluid concentration of [log] MCP-1 ([log] 0.37pg/ml/100days, SE 0.13; $p=0.0046)$, but not of other cytokines, was observed.

Conclusion: MPE fluids become less exudative and more acidic over the disease course. The rise in MCP-1 levels may suggest a pathobiological role in MPE.

\section{Words: 248}




\section{INTRODUCTION}

Malignant pleural effusion (MPE) is one of the leading causes of pleural effusions and a common cause of morbidity worldwide[1]. MPE can complicate most cancers, including extra-pleural malignancies, especially lung and breast carcinomas, as well as primary pleural mesothelioma.

The pathophysiology of MPE remains unclear but is believed to involve increased plasma extravasation from hyper-permeable tumor and/or pleural vasculature and impaired lymphatic resorption. However, no biochemical profile of the pleural fluid is pathognomonic for MPEs. The pleural fluid concentrations of protein and lactate dehydrogenase (LDH), markers of vascular permeability and pleural inflammation respectively, are usually in the exudative range by Light's criteria. Pleural fluid $\mathrm{pH}$, glucose and $\mathrm{LDH}$ levels can reflect the pleural tumor burden and its metabolic activity. Low pleural fluid $\mathrm{pH}$ and high levels of LDH have been associated with poorer survival. Lower pleural fluid $\mathrm{pH}$ also predicts MPE recurrence and clinical need for pleurodesis or indwelling pleural catheter (IPC) placement for MPE management.

MPE fluid bathes the pleural tumors and is a rich source of their secretory products including growth factors, eg vascular endothelial growth factor (VEGF), as well as cytokines/chemokines that promote tumor proliferation, eg interleukin (IL)-6[2], IL-8[3], and monocyte chemoattractant protein-1 (MCP-1)[4] etc. These molecules are present in significantly higher concentrations in the MPE than in corresponding sera, implying their local pleural synthesis. These proteins have potent biological effects and are involved in complex interplay between the host and tumor cells to execute pro-inflammatory and pro-angiogenic transcriptional programs that induce pleural inflammation, vascular hyper-permeability and tumor angiogenesis[5]. Charting the changes of these molecules during disease progression may provide clues to pleural tumor pathobiology, and may explore their potential as biomarkers of patient survival or disease activity. 
A major limitation in pleural research to date is that bio-specimens available for investigations, eg pleural fluid for biochemical and immunological profiling, are restricted to single cross-sectional samples. Repeated sampling (eg biopsies or thoracentesis) is not practical or ethical. Heterogeneity from inter-individual differences (eg genetics, cancer stages) poses major hurdles to researchers. The pleural milieu will almost certainly change throughout the disease course, and be influenced by the type, stage, progression (and treatment response) of the underlying cancer as well as host comorbidity etc.

Existing literature on the longitudinal changes in malignant pleural fluid analyses is limited to one pilot study ( $\mathrm{n}=9)$. IPC is now an established treatment for MPE[6, 7], and has been shown to provide comparable symptomatic benefits as talc pleurodesis with shorter hospitalization. The advent of IPC offers a unique opportunity, not possible before, to provide serial pleural fluid during the patients' disease course and an exciting opportunity to longitudinally study pleural cancer biology within and between individuals.

This study aimed to describe the longitudinal changes in pleural fluid biochemistry and levels of key cytokines/chemokines in patients with an MPE. 


\section{METHODS}

During the period between 01-July-2009 and 28-February-2013, 107 patients in our pleural service underwent insertion of 110 IPCs (Rocket Medical, UK) for management of MPE. It is our routine practice that patients are instructed to drain their effusion via the IPC when they are symptomatic; the frequency of drainage varied from daily to fortnightly. Most patients were followed up in the pleural service until the IPC was removed or death.

At each outpatient visit, pleural fluid samples were collected and sent for standard biochemical tests ( $\mathrm{pH}$, protein, $\mathrm{LDH}$ and glucose) and bacterial cultures. Additional fluids were centrifuged at $1020 \mathrm{~g}$ for $10 \mathrm{~min}$ at room temperature, and the supernatants were stored at $-80^{\circ} \mathrm{C}$ until assay.

All longitudinally collected pleural fluid samples were included. Most patients ( $n=87$ ) had $\geq 3$ fluid samples for longitudinal analysis of pleural fluid biochemistry; four cases had one and 12 cases had two fluid samples. In two patients with bilateral pleural effusions, fluid samples from each side were considered as separate cases. Cytokine level quantifications were performed on samples from a subset of 35 randomly selected patients from this cohort.

Results of serum biochemistry (protein, albumin, LDH and C-Reactive protein [CRP]) and hematology (hemoglobin, total white blood cell [WBC], lymphocyte, neutrophil, and platelet counts), performed for any reason, and closest to and within 14 days of pleural fluid sampling, were obtained from the hospital pathology service database.

\section{Biochemistry and hematology measurement methods}

Protein, albumin, LDH, glucose and CRP levels in blood and/or pleural fluid were measured by spectrophotometry using an Abbott Architect c16000 analyzer (Abbott Park, Illinois, USA). Pleural fluid $\mathrm{pH}$ was measured using a Radiometer ABL 800 blood-gas analyzer (Åkandevej, Brønshøj, Denmark). Hemoglobin, total white blood cells, neutrophils, 
lymphocytes, and platelets were quantified with the Beckman Coulter DxH800 analyzer (Brea, CA, USA), following standard manufacturer's instructions. All the measurements were performed at our hospital National Association of Testing Authorities-accredited laboratory.

\section{Cytokine measurement method}

Enzyme-linked immunosorbent assays (ELISAs) were used to measure concentrations of VEGF, IL-6 and IL-8 (R\&D, Minneapolis, MN), and MCP-1, IL-10, INF $\gamma$ and TNF- $\alpha$ (eBiosciences, San Diego, CA, USA). Serial samples of each patient were assayed in duplicate in the same ELISA run. The colorimetric assay was quantified by a spectrophotometer and adjusted for background.

\section{Statistical analyses}

Longitudinal changes in pleural fluid biochemistry and cytokines, serum biochemistry, and blood counts were analyzed using linear mixed models; specifically, a straight-line model with varying intercepts and varying slopes for each individual was employed. The response at the population level was modeled, either on the original scale or on a log scale, via mixed-effects linear regression using "time since first pleural fluid collection" as sole regressor. The intercepts and slopes of individuals were assumed to vary randomly about these population parameters, with this variation modeled by a bivariate normal distribution. Results reported are for the population parameters of this model.

\section{Mathematical details:}

In mathematical notation the models fitted are as follows: Let $y_{i j}$ be the response variable measured on individual $i$ at time point $t_{j}, j=1, \ldots, n_{i}$, $i=1, \ldots, n$. Here $n$ is the total number of individuals and $n_{i}$ the number of measurements taken on individual $i$, with the $j^{\text {th }}$ observation $y_{i j}$ taken at time $t_{j}$

The following linear mixed model was fitted to these data:

$$
y_{i j}=\beta_{0}+b_{0 i}+\left(\beta_{1}+b_{1 i}\right) t_{j}+\varepsilon_{i j}
$$

where $\beta_{0}$ and $\beta_{1}$ denote the population intercept and slope, respectively. 
The random effects $b_{0 i}$ and $b_{1 i}$ allow the intercept and slope of the line fitted to individual $i$ to vary about the population parameters. The joint distribution of these random effects is modeled as being a bivariate normal distribution with mean vector zero and a general variance covariance matrix. The error term, $\varepsilon_{i j}$, is assumed to be normally distributed, and independent of the random effects.

Consent was obtained from subjects to use pleural fluid and blood results for the study. The Sir Charles Gairdner Group Human Research Ethics Committee approved the study (HREC study numbers 2009-104/2020, 2011-109/2125, 2012-005/2019, 2012-038/2092 and 2012-156/2121). 


\section{RESULTS:}

\section{Demographics}

107 MPE patients underwent IPC insertions during the study period; of these pleural fluid analysis was performed in 103 patients (82\% male; 62\% had mesothelioma) (table 1). Longitudinal pleural fluid biochemistry was analyzed in 638 pleural fluid samples (from 103 patients) for protein, 627 for glucose (102 patients), 624 for LDH (103 patients) and 570 for $\mathrm{pH}$ (101 patients). The median duration between the first and final pleural fluid sampling was 95 (range 0-735) days.

Longitudinal pleural fluid cytokine testing from 35 patients was performed for MCP-1 (298 samples), VEGF (282), IL-6 (286), and IL-8 (261). IL-10, INF $\gamma$ and TNF- $\alpha$ were tested in 39 samples (5 patients) only as we found that their values were below the detection limit of the assay (IL-10 2pg/ml, INF $\gamma 4 \mathrm{pg} / \mathrm{ml}$ and TNF- $\alpha 4 \mathrm{pg} / \mathrm{ml})$. The median duration between the first and final pleural fluid sampling for this group was 148 (range 15-623) days.

\section{Pleural Fluid And Serum Biochemistry Analysis}

Pleural fluid protein, $\mathrm{pH}$, and glucose decreased while $\mathrm{LDH}$ increased over time. Pleural fluid protein (intercept 40.97g/L; $n=638$ ) decreased by 8g/L/100days (SE 1.32; $p<0.0001$ ), Figure 1. Pleural fluid $\mathrm{pH}$ (intercept 7.34; $\mathrm{n}=570$ ) decreased by 0.04/100days (SE 0.02; $p=0.0203$ ), Figure 2, whereas pleural fluid glucose (intercept, 3.84mmol/L; $\mathrm{n}=627$ ) showed a non-significant decrease by $0.07 \mathrm{mmol} / \mathrm{L} / 100$ days (SE, $0.12 ; p=0.4855$ ). On the other hand, the pleural fluid LDH [log] values (intercept, $6.29[\log ] \mathrm{U} / \mathrm{L}$; $\mathrm{n}=624$ ) increased by 0.08 [log]U/L/100days (SE 0.05; $p=0.0787$ ).

\section{Serum albumin significantly decreased with time. Serum protein} decreased less than the corresponding pleural fluid protein reduction. Both serum protein (intercept 70.58g/L; SE 0.75; $\mathrm{n}=364$ ) and albumin (intercept $38.35 \mathrm{~g} / \mathrm{L} ; \mathrm{n}=384$ ) decreased over time by $0.75 \mathrm{~g} / \mathrm{L} / 100$ days (SE $0.47 ; p=0.1068$ ) and $3 \mathrm{~g} / \mathrm{L} / 100$ days (SE $0.51 ; p<0.0001$ ) respectively.

Subgroup analysis of 364 samples where both pleural fluid and corresponding serum protein results were available showed that reduction of 
protein was greater in the pleural fluid compared to its corresponding serum level. The pleural fluid protein (intercept 39.85g/L) decreased by $5 \mathrm{~g} / \mathrm{L} / 100$ days (SE 1.22; $p<0.0001$ ) whereas the pleural fluid to serum protein ratio (intercept 0.56; SE 0.02) decreased by 0.06/100 days (SE 0.02; $p=0.04$ ). Serum CRP levels did not change significantly.

\section{Malignant pleural effusions from mesothelioma vs non-mesothelioma} patients: MPEs from mesothelioma had significantly lower baseline pleural fluid glucose ( $p=0.0104 ; n=63)$ compared to non-mesothelioma $(n=38)$. A positive difference in slopes for these two groups of patients ( $p=0.0423$ ) was seen with pleural fluid glucose, but not others, that suggested the reduction in glucose is more in the non-mesothelioma group. The significance of this finding is not clear.

\section{Pleural Fluid Cytokine Analysis}

Pleural fluid MCP-1, but not any of the other tested cytokines, increased significantly over time. Pleural fluid [log] MCP-1 (intercept, [log] $6.72 \mathrm{pg} / \mathrm{ml} ; \mathrm{n}=298$ ) increased by $[\mathrm{log}] 0.37 \mathrm{pg} / \mathrm{ml} / 100$ days (SE 0.13; $p=0.0046$ ), Figure 3. Levels of [log] VEGF level (intercept [log] 6.29 pg/ml; $\mathrm{n}=282$ ) increased by [log] 0.11pg/ml/100 days (SE 0.07; $p=0.14$ ), [log] IL-6 (intercept [log] $6.52 \mathrm{pg} / \mathrm{ml} ; \mathrm{n}=286$ ) decreased by [log] $0.05 \mathrm{pg} / \mathrm{ml} / 100$ days (SE 0.10; $p=0.64$ ), and [log] IL-8 (intercept [log] 5.61pg/ml; $\mathrm{n}=261$ ) decreased by [log] $0.07 \mathrm{pg} / \mathrm{ml} / 100$ days (SE 0.09; $p=0.42$ ).

\section{Blood hematology analysis}

Hemoglobin and lymphocyte counts decreased significantly; platelets increased significantly. Hemoglobin (intercept 133.78g/L) decreased by 13g/L/100 days (SE 2.11; p-value, <0.0001) in the 480 samples tested. Lymphocytes count (intercept $1.28 \times 10^{9} / \mathrm{L} ; \mathrm{n}=457$ ) decreased by $0.07 \times 10^{9} / \mathrm{L} / 100$ days (SE 0.02; $p=0.0012$ ). Platelets (intercept 299x10 $/ \mathrm{L}$ ) increased significantly by $26 \times 10^{9} / \mathrm{L} / 100$ days (SE 6.32; $p<0.0001$ ). No significant increase in leukocyte and neutrophil counts was observed. 


\section{DISCUSSION}

Pleural fluid bathes the pleura and its importance in providing diagnostic clues (eg Light's Criteria, fluid cytology etc.) is well recognized. Previous studies on pleural fluids mainly contain cross-sectional data. The advent of IPC provides this unique opportunity for us to report the longitudinal changes in pleural fluid composition and cytokines in a sizeable cohort of MPEs. Our data show that pleural fluid $\mathrm{pH}$ decreased significantly over time while LDH trended upwards. The protein levels remained stable in the blood but decreased in the MPE. The pleural fluid concentration of MCP-1, a cytokine recently shown to play an important role in exudative effusion formation, rose significantly with advanced stage tumor.

The longitudinal reduction in pleural fluid $\mathrm{pH}$ and a corresponding rise in $\mathrm{LDH}$ were in keeping with increased metabolic activity within the pleural cavity. Low pleural fluid $\mathrm{pH}$ is known to reflect greater pleural tumor burden, and has been associated with a poorer prognosis and shorter survival in MPE patients.[8-10] Our finding that pleural fluid $\mathrm{pH}$ continued to decrease with progression of pleural malignancy over time further support a relationship between the effusion $\mathrm{pH}$ and the cancer load in the pleura.

Pleural fluid glucose, like $\mathrm{pH}$, has been associated with advanced pleural cancers. However, the glucose level in pleural fluid is strongly influenced by its corresponding blood concentration that in turn can be affected by concurrent diabetes and other illnesses causing hyperglycemia. This may explain the absence of any consistent trend of pleural fluid glucose levels over time.

The protein concentrations in the pleural fluid decreased over time while its levels remained stable in the serum. It is noteworthy that repeated removal of exudative malignant fluid via IPC drainage did not result in noticeable reduction in systemic protein levels, a concern that has been raised regarding IPC use. Pleural fluid protein is generally regarded as a marker of plasma leakage from vascular hyper-permeability in the pleura[5]. Pleural 
fluid protein level in the exudative range can be found in more than 60 diseases, and is a key mechanism underlying malignant effusion formation. Plasma leakage in MPE is believed to be driven by mechanisms induced by local pleural tumor and substances released by it. No data exist on longitudinal measurements of effusion protein but it would be expected to rise with progression of pleural malignancy; our data showed otherwise. We hypothesize that reduction in pleural fluid protein level results from concurrent driving forces of formation of low protein pleural fluid, such as heart failure, presence of trapped lung, and low systemic protein levels from cancer-associated malnutrition. Our data suggests that the final pleural fluid protein levels would likely not have fulfilled the exudate criteria; however, the fluid would fulfill Light's criteria for exudate as the LDH levels remained high.

Our data showed that serum albumin, but not protein, level decline longitudinally in this cohort.

The pathobiology of exudative pleural effusion (including MPE) formation is poorly understood. Many cytokines/biological mediators are no doubt involved in the pleural inflammation, and vascular hyper-permeability processes that underlie plasma extravasation. Detailing their longitudinal changes may provide hints on the key drivers of the fluid formation process. A pilot study of 6 cases demonstrated that pleural fluid transforming growth factor-b1 (TGF- b1) concentration in MPE increased significantly 2 weeks after IPC insertion compared to the level at time of IPC insertion. Plasminogen activator inhibitor-1 and vascular endothelial growth factor levels did not show a significant increase. Of the large range of mediators we tested, MCP-1 rose with time (and tumor progression) in MPE. MCP-1 was the only cytokine to demonstrate a consistent trend. This finding is timely as recent pre-clinical studies point towards MCP-1 playing a crucial role in exudative and malignant effusion formation.

MCP-1 was highly expressed by lung cancer cells in a mouse model, and induced vascular permeability and fluid leak. Antagonists of MCP-1 significantly reduced MPE formation in mice with lung cancers[11]. This 
finding was corroborated in a separate study on the effects of intrapleural fibrinolytics (especially tissue plasminogen activator [tPA]), which consistently induced accumulation of large volume of pleural exudative fluid[12]. Lansley et al also found that MCP-1 level correlated with fluid volume and strategies to antagonize MCP-1 (eg using soluble antibodies and receptor antagonists) all significantly reduced exudate formation. Our study lends important human data to support these pre-clinical studies that MCP-1 may be a critical target in future therapy for MPE control.

Our study has limitations. First, it was a retrospective analysis although most patients included had complete pleural fluid data. Second, there was a relatively high proportion of mesothelioma in this cohort due to the high prevalence of mesothelioma in Western Australia. Patients with mesothelioma also had a higher number of longitudinal samples as they were more likely to survive and have IPC in-situ longer compared to metastatic carcinomas. Third, we accepted data from corresponding blood drawn within 14 days of pleural fluid sampling. However, the majority of paired samples were collected within 3 days of each other (49\% on the same day and $70 \%$ within 3 days). Third, in the mathematical modeling, the use of log-transformation was based mainly on the range of that response variable and the amount of skewness visible in the (marginal) histograms of these variables. Log-transformation can vary the interpretation of the regression parameters. Finally, our linear mixed model assumed an independent and identically distributed residual error structure.

This sizable longitudinal study describes the changes in pleural fluid compositions over time, and found that the pleural fluid protein and $\mathrm{pH}$ decreased while MCP-1 level increased. Serum protein does not reduce significantly even when the malignant effusion is drained regularly. These data provide an important platform for future studies in the field. 


\section{REFERENCES}

1. Lee, Y.C. and R.W. Light, Management of malignant pleural effusions. Respirology, 2004. 9(2): p. 148-56.

2. Yeh, H.H., et al., Autocrine IL-6-induced Stat3 activation contributes to the pathogenesis of lung adenocarcinoma and malignant pleural effusion. Oncogene, 2006. 25(31): p. 4300-4309.

3. Ceyhan, B.B., et al., IL-8 in pleural effusion. Respir Med, 1996. 90(4): p. 215-21.

4. Stathopoulos, G.T., et al., A Central Role for Tumor-derived Monocyte Chemoattractant Protein-1 in Malignant Pleural Effusion. Journal of the National Cancer Institute, 2008. 100(20): p. 14641476.

5. Stathopoulos, G.T. and I. Kalomenidis, Malignant pleural effusion: tumor-host interactions unleashed. Am J Respir Crit Care Med, 2012. 186(6): p. 487-92.

6. Davies, H.E., et al., Effect of an indwelling pleural catheter vs chest tube and talc pleurodesis for relieving dyspnea in patients with malignant pleural effusion: the TIME2 randomized controlled trial. JAMA, 2012. 307(22): p. 2383-9.

7. $\quad$ Fysh, E.T., et al., Indwelling pleural catheters reduce inpatient days over pleurodesis for malignant pleural effusion. Chest, 2012. 142(2): p. 394-400.

8. Aelony, Y., J.F. Yao, and R.R. King, Prognostic value of pleural fluid $\mathrm{pH}$ in malignant epithelial mesothelioma after talc poudrage. Respiration, 2006. 73(3): p. 334-9.

9. Rodriguez-Panadero, F., F. Borderas Naranjo, and J. Lopez Mejias, Pleural metastatic tumours and effusions. Frequency and pathogenic mechanisms in a post-mortem series. Eur Respir J, 1989. 2(4): p. 366-9.

10. Sahn, S.A. and J.T. Good, Jr., Pleural fluid $p H$ in malignant effusions. Diagnostic, prognostic, and therapeutic implications. Ann Intern Med, 1988. 108(3): p. 345-9.

11. Stathopoulos, G.T., et al., A central role for tumor-derived monocyte chemoattractant protein-1 in malignant pleural effusion. J Natl Cancer Inst, 2008. 100(20): p. 1464-76.

12. Lansley, S.M., et al., Tissue Plasminogen Activator Potently Stimulates Pleural Effusion via a Monocyte Chemotactic Protein-1Dependent Mechanism. Am J Respir Cell Mol Biol, 2015. 53(1): p. 105-12. 


\section{Figure Legends:}

Figure 1 - Shows longitudinal changes in pleural fluid protein analyzed using linear mixed model. Pleural fluid protein (intercept $40.97 \mathrm{~g} / \mathrm{L} ; \mathrm{n}=638$ ) decreased by 8g/L/100days (SE 1.32; $p<0.0001$ ).

Each dot represents an individual fluid result and each color represents an individual patient. The central black line represents the progression of the particular parameter over time in a typical individual from the population, according to the mixed model fitted to the data. The dark shaded band represents the (point wise) 95\% confidence interval around the fitted response curve at the population level. The light shaded band represents the (point wise) 95\% prediction interval around the fitted response curve at the population level.

Figure 2 - Shows longitudinal changes in pleural fluid $\mathrm{pH}$ analyzed using linear mixed model. Pleural fluid $\mathrm{pH}$ (intercept 7.34; $\mathrm{n}=570$ ) decreased by 0.04/100days (SE 0.02; $p=0.0203$ ).

Figure 3 - Shows longitudinal changes in pleural fluid MCP-1 analyzed using linear mixed model. Pleural fluid log (MCP-1) (intercept, 6.72pg/ml; $\mathrm{n}=298$ ) increased by $0.37 \mathrm{pg} / \mathrm{ml} / 100$ days (SE 0.13; $p=0.0046$ ). 
Table 1: Demographic characteristics of longitudinal pleural fluid cohort Total number of cases $=103$

\begin{tabular}{|l|l|}
\hline Male: $\mathrm{n}(\%)$ & $82(80 \%)$ \\
\hline Age: median (range) & 63 years (53-83) \\
\hline Cancer type: & \\
Mesothelioma & $63(61 \%)$ \\
Lung cancer & $16(16 \%)$ \\
Breast cancer & $6(5 \%)$ \\
Others & $18(18 \%)$ \\
& \\
\hline No. of cases with & \\
1 fluid sample & 4 \\
2 & 12 \\
$3-5$ & 41 \\
$6-10$ & 31 \\
$>10$ & 15 \\
\hline
\end{tabular}

Additional column for cytokines

Male: 32

Age: 69 (52-89)

Cancer type: Mesothelioma

Fluid samples:

$1-5 ; 2-1 ; 3-5-12 ; 6-10-7 \&>10-10$ 


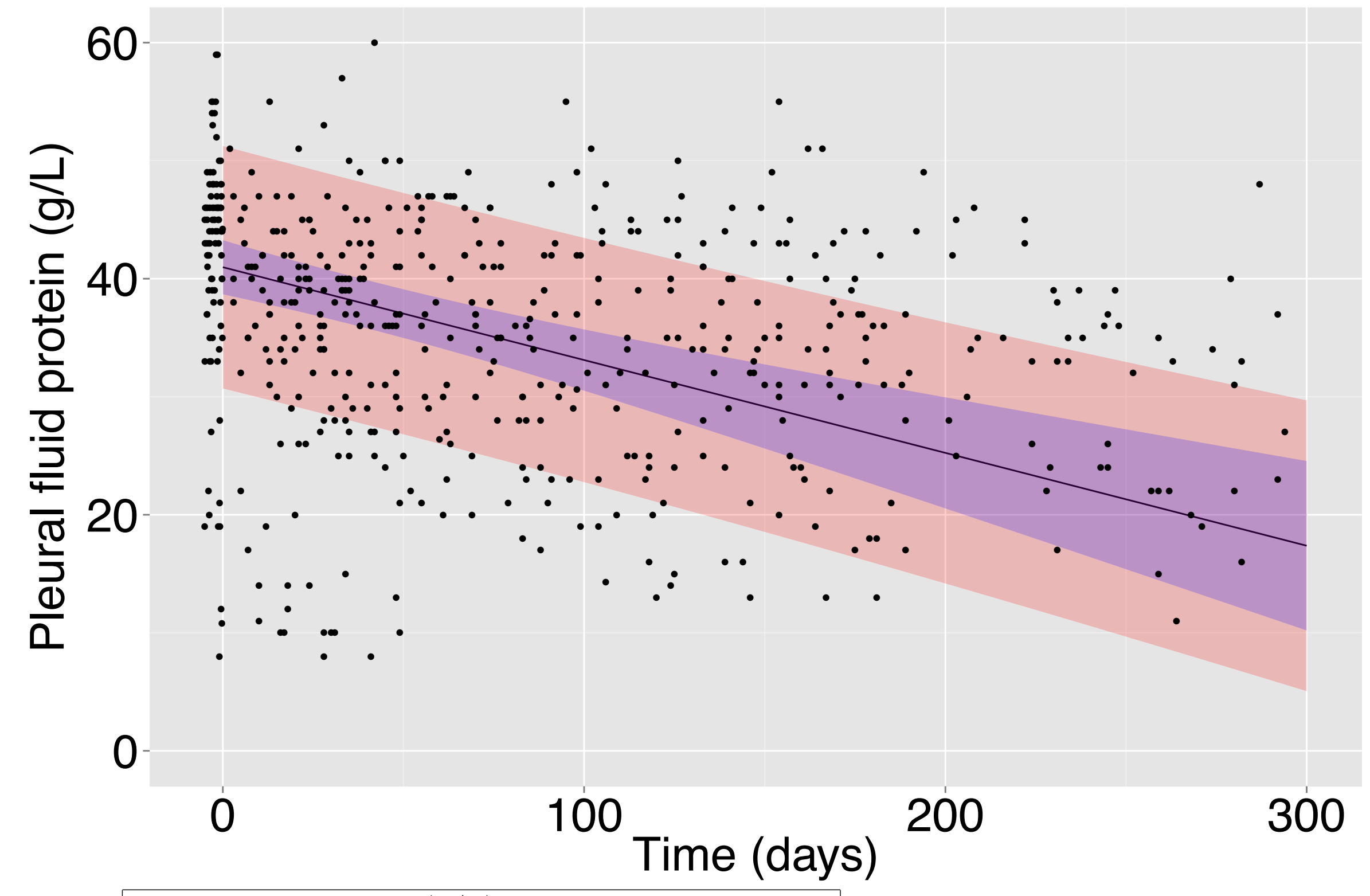

Pleural fluid protein (g/L) vs 100 Days

Value Std.Error p-value

$\begin{array}{llll}\text { (Intercept) } & 40.97 \quad 1.15<0.0001\end{array}$

$\Delta / 100$ Days $\quad-8 \quad 1.32<0.0001 \quad n=638$ 


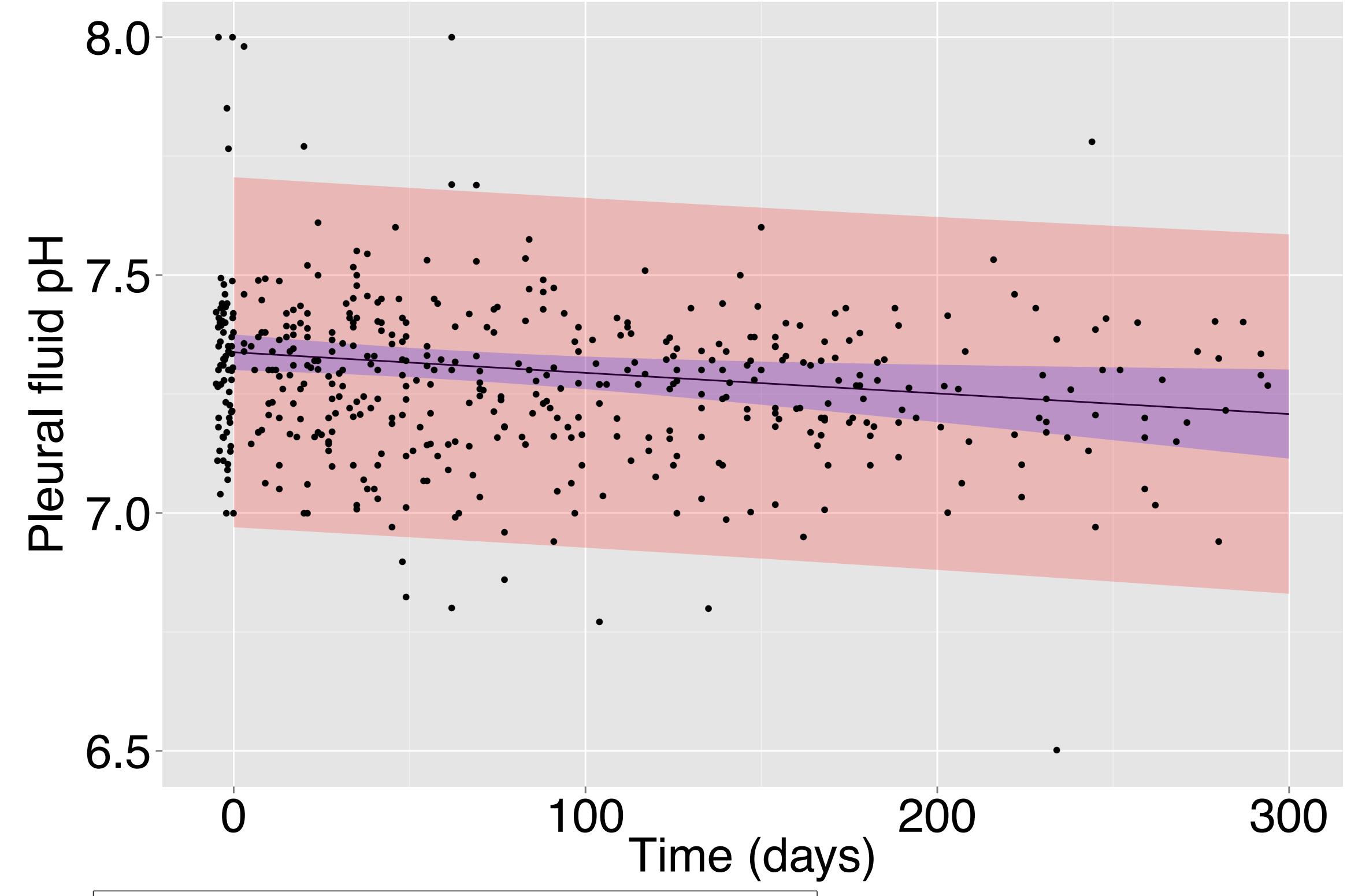

\begin{tabular}{|rrrrr|}
\hline \multicolumn{4}{|c|}{ Pleural fluid $\mathrm{pH}$ vs 100 Days } & \\
& Value & Std.Error & p-value & \\
(Intercept) & 7.34 & 0.02 & $<0.0001$ & \\
$\Delta / 100$ Days & -0.04 & 0.02 & $0.0203 \quad n=570$ \\
\hline
\end{tabular}




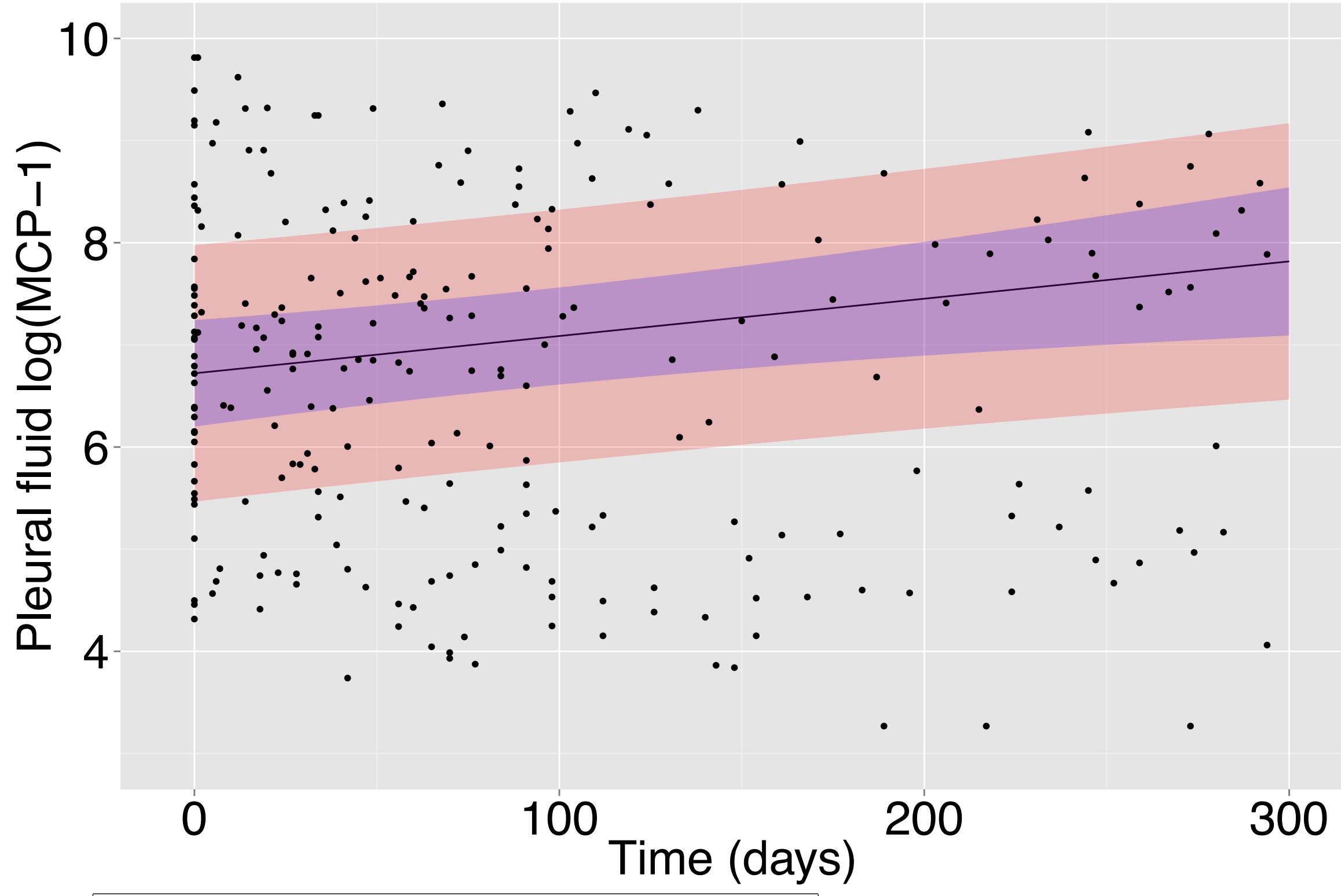

\begin{tabular}{|rrrr|}
\hline Pleural fluid $\log (\mathrm{MCP}-1)$ & $(\mathrm{pg} / \mathrm{ml})$ vs 100 Days \\
& Value & Std.Error & p-value \\
(Intercept) & 6.72 & 0.26 & $<0.0001$ \\
$\Delta / 100$ Days & 0.37 & 0.13 & $0.0046 \quad n=298$ \\
\hline
\end{tabular}

